\title{
Management of proximal interphalangeal joint injuries
}

Arnis Freiberg MD FRCSC FACS

\begin{abstract}
A Freiberg. Management of proximal interphalangeal joint injuries. Can J Plast Surg 2007;15(4):199-203.

Injuries to the proximal interphalangeal joint are common but frequently missed. They are often overtreated by prolonged immobilization, resulting in stiffness, which may be permanent. The purpose of the present article is to briefly review the relevant anatomy and biomechanics, present an approach to physical examination and diagnosis, and propose a practical clinical classification. The treatment of the most severe injury - the intra-articular fracture dislocation - is controversial. The various treatment options are discussed, based on personal experience and a review of the literature. A list of relevant references is presented.
\end{abstract}

Key Words: Management; Proximal interphalangeal joint injuries; Review of literature

\section{La prise en charge des lésions des articulations interphalangiennes proximales}

Les lésions des articulations interphalangiennes proximales sont courantes, mais passent souvent inaperçues. Par ailleurs, elles sont souvent trop traitées par une immobilisation prolongée, ce qui provoque une raideur qui risque de devenir permanente. Le présent article vise à analyser brièvement l'anatomie et la biomécanique pertinentes, à présenter un mode d'examen physique et de diagnostic et à effectuer une classification clinique pratique. Le traitement de la lésion la plus grave, la fracture-luxation intra-articulaire, est controversé. On expose les diverses possibilités de traitement, d'après l'expérience personnelle et une analyse bibliographique. On présente également une liste de références pertinentes.

The low-velocity injuries are less common and are usually caused by twisting; this often causes disruption of the thin and filmy volar plate origin.

\section{CLINICAL EXAMINATION AND DIAGNOSIS}

At the time of the injury

A complete and thorough examination is quite often not possible because of swelling and pain, as seen in the more severe injuries. In less severe injuries, point tenderness and localized swelling quite accurately indicates the site of injury. Testing for joint stability at this point in time is not very important and can be deferred until the next early postinjury visit. Plain $\mathrm{x}$-rays are valuable for two reasons:

- to diagnose a complete dislocation or extent of subluxation; and

- to look for avulsion fractures. Unless the fragment is large, the fracture itself is not important, but it will indicate the site of the ligamentous injury.

Late (one week or more after the time of injury)

Test joint stability gently to prevent further damage to the partially torn ligaments. Next, look for areas of point tenderness at the following six sites:

- insertion and origin of the ulnar and radial collateral ligaments;

- insertion and origin of the volar plate; and

- insertion of the central slip. 
The ideal time for this examination is approximately seven to 10 days following the injury.

Later, once the soft tissues have partially healed, the exact diagnosis may be more difficult. A correct and precise diagnosis is important in choosing the most appropriate treatment (eg, for injuries such as avulsion of the central slip insertion, splinting as opposed to early motion is performed to prevent a late boutonniere deformity). Similarly, it is important to identify the site of the collateral ligament injury to choose the correct digit for buddy-taping.

\section{IMAGING}

Plain radiographs are sufficient in supporting the clinical diagnosis. It is important to order not only the standard anteroposterior and lateral views, but also two oblique views to properly identify small avulsion fractures. Although small avulsion fractures themselves are not important, they will identify the sites of the ligament injuries.

For larger bony fragments, it is important to assess not only the size of the avulsed fragment, but also the degree of displacement or rotation. A good lateral view is essential in identifying the degree of subluxation or dislocation.

It is also important to order postreduction films for two reasons:

- to prove and document a successful reduction; and

- for medicolegal reasons.

\section{CLASSIFICATION}

Although there are several classifications described in the literature, I prefer a rather simple classification:

- dislocations - volar, dorsal;

- subluxations - volar, dorsal, ulnar, radial;

- avulsion or 'chip' fractures associated with ligamentous injuries; and

- intra-articular fractures or fracture dislocations:

- unipolar - proximal phalanx: one condyle, two condyles or comminuted; middle phalanx: comminuted;

- bipolar: complete joint destruction.

\section{DISLOCATIONS AND SUBLUXATIONS}

Most dislocations are closed and are easily reduced when closed. Compound or open dislocations are usually volar and often irreducible closed, requiring open reduction.

\section{Volar dislocations}

Volar dislocations are the least common, are often most difficult to reduce and carry a poorer prognosis. Compound dislocations may require open reduction, because either one or both condyles can be caught between the flexor tendons and the flexor tendon sheath. The reduction is usually carried out under digital block anesthesia.

Following reduction, buddy-taping and early motion is usual. An extension block splint may be necessary in unstable cases.

\section{Dorsal dislocations}

Dorsal dislocations are the most common, are usually closed and easily reduced closed. Open reduction may be required in cases of volar plate entrapment. The postreduction routine is the same as in volar dislocations.

\section{Subluxations}

Depending on the degree, reduction may not be necessary. In most cases, buddy-taping is the only postinjury care needed; the appropriate digit depends on the site of injury (ulnar or radial). Poorest end results are seen in injuries to the little finger because of the length discrepancy between the little and ring fingers.

Splinting of the PIP joint at various angles is usually performed by emergency physicians and, if not seen early on, may lead to permanent stiffness. It is therefore important to see these patients as soon after the injury as possible. This requires educating the emergency physicians and screening of referrals to the hand clinics.

It is my firm belief that even a healthy, young, uninjured PIP joint can only be safely totally immobilized for two weeks. If one adds age, injury and type of finger to this equation, the safe immobilization time is even shorter.

Early protected motion is therefore the key to a reasonably successful outcome. It is also very important to warn the patient at the time of the initial visit that, in general terms, injuries to the PIP joint are serious and the final outcome will most likely result in a less-than-normal-looking and -functioning digit. It is the patient's - often erroneous - perception that a simple 'jammed' digit, often reduced at the time of injury, may lead to serious consequences. It is also important to explain to the patient that there is a lot of hard work ahead as far as rehabilitation and hand therapy are concerned. The patient must be warned that the therapy will be painful and reminded of the old slogan seen in hand therapy departments: 'no pain, no gain'. A prescription for an analgesic to be taken before the therapy will be appreciated by the patient.

It is interesting to note that one rarely sees unstable PIP joints, with the exception of individuals with congenitally hyperextensile joints. I believe that for any one unstable joint, there are at least 100 permanently stiff PIP joints!

Therefore, early motion is the key to a successful outcome. To accomplish this, I prefer to see these patients as soon as possible postinjury and follow them at weekly intervals.

\section{INTRA-ARTICULAR FRACTURE DISLOCATIONS}

\section{Treatment options}

Because there are a number of well-recognized treatment options, it is obvious that there is not one method superior to another. Unfortunately, there are no prospective or controlled studies available, and as far as I can see, there will not be any in the foreseeable future. Because of the diversity of the various types of injury and digits involved, one would need a large number of cases to satisfy statistical requirements.

Long-term follow-up may also be difficult, because these injuries are more common in the young, mobile population. Each digit presents with specific problems - especially the little finger. Last but not least, the patient's occupation and degree of motivation have to be taken into account, as well as the availability and skill of the hand therapist, who is of utmost importance for postinjury care.

Finally, there is the problem of a universally accepted classification of these injuries. A rather comprehensive and practical classification was presented by Schenck in 1994 (3); 
however, a review of several articles dealing with various treatment method preferences found their authors preferred to use their own classifications, making outcome comparisons almost impossible.

During the past 20 years (or longer), I have used a simple and practical clinical classification for intra-articular fractures:

- unipolar;

- bipolar; and

- complete joint destruction or comminuted bipolar fractures (the following subclassification deals with the size of the fracture fragment):

- large - one that is large enough for fixation either by a pin or a lag screw, usually at the base of the proximal phalanx, or one or two condyles; and

- small - not suitable for fixation, usually involving the head of the middle phalanx or both of the above (complete joint destruction).

The large fragment may be displaced, undisplaced or rotated.

Early motion and buddy-taping: My preferred treatment method of management is early motion and buddy-taping, with or without extension block splinting.

The rationale for this method is that all fractures eventually heal, but most, if not moved early, become stiff, some permanently.

In addition, the PIP joint has very limited space, and as long as the enclosing soft tissue 'envelope' has not been severely disrupted, early motion realigns the small fracture fragments in a nearly normal anatomical position.

The basic principles are twofold:

- maintain concentric reduction of the joint; and

- institute early motion to minimize or prevent stiffness.

Buddy taping is most successful in digits 2 to 4 . Although the little finger is one the more commonly injured digits, because of length discrepancy between digit 4 and digit 5 , the final results are inferior compared with the other digits.

If the fracture requires reduction and fixation, these are carried out at the time of the injury, and buddy taping started immediately after.

Indications for reduction: There are very very few indications for open reduction. They include:

- unstable or rotated condylar fractures;

- irreducible, usually volar, fracture dislocations; and

- volar plate entrapment.

Contrary to some surgeons' opinions, collateral ligaments do not need to be surgically repaired. My preference for operative exposure is a dorsal, longitudinal, midline incision that can be extended, if necessary, from the distal interphalangeal joint to the metacarpophalangeal joint.

Crossing a joint at right angles is only contraindicated on the volar surface. I have not encountered any problems using this incision on the dorsal surface. Furthermore, a midline incision avoids injury to the dorsal sensory branches.

Indications for extension block splinting: After reduction or fixation, the joint is tested in flexion and extension. We know that approximately $90 \%$ of finger fractures are stable in flexion; therefore, one must note the degree of extension at which the reduction becomes unstable. The splint is then designed to block at this angle. If there is no loss of fixation at maximum extension, the splint is not necessary. Weekly follow-up is necessary to make sure that the patient is following instructions and has not lost the reduction. Referral to a hand therapist is only needed if the patient has problems with the splint, needs assistance in buddy taping or shows lack of motivation.

\section{Personal experience and comments}

With a few exceptions, I have been using this approach for most intra-articular fractures over the past 20 years. Although I do not have a large number of cases with long-term follow-up, I have been generally quite satisfied with the results.

It is interesting to note the similarity of the radiological appearance over one year following injury, especially in cases involving the base of the middle phalanx.

The base of the middle phalanx remodels to a shape similar to an inverted umbrella. In general terms, very few patients complain of pain or instability. It is also interesting to note that post-traumatic arthritis is very rare in fractures confined to the base of the middle phalanx. The range of motion is also usually functionally quite satisfactory. We have always stressed the importance of flexion over extension in digits 4 and 5 and extension over flexion in digits 2 and 3. In general terms, final results are better in digits 2 and 3 than in digits 4 and 5 because of the length discrepancy between digits 4 and 5 during buddytaping.

\section{Advantages and disadvantages of early motion and buddy-taping \\ Advantages:}

- simplicity;

- limited need for an experienced hand therapist;

- absence of bulky splints; and

- more suitable for cold climates - with the exception of extension block splints, the patient is able to wear mitts during cold weather.

Disadvantages: Because of the absence of traction, foreshortening of the digit is not prevented. However, because the most commonly injured digit is the little finger, slight foreshortening is functionally not disabling. Patient compliance and motivation, or lack thereof, are most likely no different than with other treatment methods. Pain and discomfort during rehabilitation may be less when using distraction methods.

Again, it must be pointed out that it is almost impossible to compare this method with the others.

\section{ALTERNATIVE METHODS OF TREATMENT}

\section{The dynamic traction method}

This method combines movement and traction, thus preventing foreshortening while maintaining mobility. The motion can be either active or passive, and there are several modifications described in the literature. The procedure was first described by Schenck in 1986 (15). A comprehensive review was presented by Schenck in 1994 (16). Various splinting techniques have been nicely presented by hand therapists Kearney and Brown (4). 
Because I decided early on in my practice to treat all PIP joint fracture dislocations by buddy-taping and early motion, I have no experience with this method. On reviewing the literature, it appears to be a popular and well-accepted treatment method.

\section{Advantages:}

- combination of traction and motion;

- choice between active and passive motion;

- choice between continuous and intermittent motion;

- reduction and realignment of articular fragments; and

- distal distraction force, which prevents or minimizes contractures of ligaments and periarticular structures.

\section{Disadvantages:}

- requires the services of a trained hand therapist;

- the splint is bulky - not suitable for use in cold environments;

- cost; and

- patient understanding of the therapy protocol may be poor.

\section{Closed reduction and percutaneous $\mathrm{K}$-wire fixation Indications:}

- relatively large fracture fragments; and

- lack of comminution of fracture fragments.

\section{Advantages:}

- reduced scarring and subsequent stiffness;

- semi-rigid fixation; and

- avoidance of added injury to the soft tissue 'envelope'.

\section{Disadvantages:}

- need for an image intensifier;

- possible pin tract infection; and

- possible need for a second procedure for K-wire removal.

\section{Open reduction and internal fixation}

\section{Indications:}

- inability to obtain adequate reduction or fixation by percutaneous $\mathrm{K}$-wire fixation;

- relatively large fracture fragments;

- absence of comminution; and

- rotated condylar fractures.

\section{Advantages:}

- ability to obtain anatomical reduction; and

- stable fixation.

\section{Disadvantages:}

- increased soft tissue scarring predisposing to stiffness; and

- technically demanding.

Volar plate arthroplasty

First described and popularized by Eaton (21), volar plate arthroplasty is indicated for reconstruction of a unipolar, comminuted fracture, usually at the base of the middle phalanx.

\section{Advantages:}

- uses autogenous material;

- maintains length;

- may be used as a primary or secondary procedure; and

- resurfaces the damaged articular surface.

\section{Disadvantages:}

- technically demanding;

- requires relatively extensive soft tissue dissection, hence potentially more scarring;

- potential instability;

- may produce an angular deformity;

- precludes early motion;

- may require removal of the $\mathrm{K}$-wire or pull-out wire; and

- potential K-wire infections.

Interpositional arthroplasty - silicone This technique was first described and popularized by Swanson and de Groot Swanson (20).

\section{Advantages:}

- most suitable as a salvage procedure for secondary reconstruction;

- maintains some movement;

- eliminates or minimizes pain; and

- maintains length.

\section{Disadvantages:}

- requires extensive soft tissue dissection, therefore predisposing to postoperative stiffness;

- technically demanding;

- more expensive than other methods;

- precludes the use of possible late arthrodesis;

- not indicated for the index finger - not strong enough for power pinch; and

- possible implant breakage and subsequent silicone synovitis. 


\section{Arthrodesis}

Arthrodesis is most commonly used as a salvage procedure.

\section{Advantages:}

- stability - especially indicated in the index finger; and

- eliminates pain.

\section{Disadvantages:}

- loss of movement;

- some loss of length;

- poor choice for little and ring fingers, where flexion is important; and

- difficulty in determining the ideal angle of fusion, especially digits 4 and 5 .

\section{SUMMARY AND CONCLUSIONS}

Injuries to the PIP joint are serious and must be treated with respect. In addition, expectations and outcomes must be discussed with the patient. Emergency physicians must be made aware of the possible sequelae and educated in making an accurate diagnosis as well as the need for an early referral to a hand surgeon, to hopefully avoid potential complications. Surgeons must realize that the main problem in treating these injuries is to avoid permanent deformity and stiffness. Correct initial diagnosis is of utmost importance in choosing the most successful treatment plan. It must be remembered that most of the errors are committed by overtreatment rather than undertreatment! Open reduction and internal fixation predisposes to stiffness and should be avoided, if possible. There is no need for repair of collateral ligaments. Because there are many treatment choices available for treating severe intra-articular fracture dislocations and no reliable, scientifically proven studies, the surgeon must use the method with which he or she is most familiar and comfortable.

Unfortunately, well-controlled, outcome-based studies will not be forthcoming in the near future because of the relatively small number of cases available, the diversity of digits involved, injury patterns, lack of a universally accepted classification and outcome results. A multicentre project may provide the answer.

\section{REFERENCES AND SELECTED BIBLIOGRAPHY}

Anatomy and Physiology

1. Leibovic SJ, Bowers WH. Anatomy of the proximal interphalangeal joint. Hand Clin 1994;10:169-78.

2. Salter RB. The physiologic basis of continuous passive motion for articular cartilage healing and regeneration. Hand Clin 1994;10:211-9.

Classification

3. Schenck RR. Classification of fractures and dislocations of the proximal interphalangeal joint. Hand Clin 1994;10:179-85.
Treatment - General

Hand therapists management

4. Kearney LM, Brown KK. The therapist's management of intraarticular fractures. Hand Clin 1994;10:199-209.

General

5. McPhee SD. Extension block splinting for the proximal interphalangeal joint. Am J Occup Ther 1987;41:389-90.

6. Freiberg A, Pollard BA, Macdonald MR, Duncan MJ. Management of proximal interphalangeal joint injuries. J Trauma 1999;46:523-8

7. Chinchalkar SJ, Gan BS. Management of proximal interphalangeal joint fractures and dislocations. J Hand Ther 2003;16:117-28.

Surgical management

8. Lubahn JD. Dorsal fracture dislocations of the proximal interphalangeal joint. Hand Clin 1988;4:15-24.

9. Majumder S, Peck F, Watson JS, Lees VC. Lessons learned from the management of complex intra-articular fractures at the base of the middle phalanges of fingers. J Hand Surg (Br) 2003;28:559-65.

10. Kang R, Stern PJ. Fracture dislocations of the proximal interphalangeal joint. J Am Soc Surg Hand 2002;2:47-59.

Specific methods of management

Extension block splinting

11. Dobyns JH, McElfresh EC. Extension block splinting. Hand Clin 1994;10:229-37.

12. Lopez MS, Hanley KF. Dynamic extension block splinting for proximal interphalangeal joint injuries. Mil Med 1985;150:423-6.

Closed reduction and K-wire fixation

13. Newington DP, Davis TR, Barton NJ. The treatment of dorsal fracture-dislocation of the proximal interphalangeal joint by closed reduction and Kirschner wire fixation: A 16-year follow up. J Hand Surg (Br) 2001;26:537-40.

14. Aladin A, Davis TR. Dorsal fracture-dislocation of the proximal interphalangeal joint: a comparative study of percutaneous Kirschner wire fixation versus open reduction and internal fixation. J Hand Surg (Br) 2005;30:120-8.

Dynamic traction method

15. Schenck RR. Dynamic traction and early passive movement for fractures of the proximal interphalangeal joint. J Hand Surg (Am) 1986;11:850-8

16. Schenck RR. The dynamic traction method. Combining movement and traction for intra-articular fractures of the phalanges. Hand Clin 1994;10:187-98.

17. Badia A, Riano F, Ravikoff J, Khouri R, Gonzalez-Hernandez E, Orbay JL. Dynamic intradigital external fixation for proximal interphalangeal joint fracture dislocations. J Hand Surg (Am) 2005;30:154-60.

Open reduction and internal fixation

18. Freeland AE, Benoist LA. Open reduction and internal fixation method for fractures at the proximal interphalangeal joint. Hand Clin 1994;10:239-50.

Arthroplasty

19. Kobayashi Ky, Torrono AL. Proximal interphalangeal joint arthroplasty of the hand. J Am Soc Surg Hand 2003:3:219-26

20. Swanson AB, de Groot Swanson G. Flexible implant resection arthroplasty of the proximal interphalangeal joint. Hand Clin 1994;10:261-6.

Volar plate arthroplasty

21. Malerich MM, Eaton RG. The volar plate reconstruction for fracture-dislocation of the proximal interphalangeal joint. Hand Clin 1994;10:251-60.

Arthrodesis

22. Jones BF, Stern P. Interphalangeal joint arthrodesis. Hand Clin 1994;10:267-75. 